\title{
Solitons in a one-dimensional interacting Bose-Einstein system
}

\author{
R K Bhaduri ${ }^{1}$, Sankalpa Ghosh ${ }^{2}$, M V N Murthy ${ }^{2}$ and Diptiman Sen ${ }^{3}$ \\ ${ }^{1}$ Department of Physics and Astronomy, McMaster University, Hamilton, Ontario, Canada L8S \\ $4 \mathrm{M} 1$ \\ 2 The Institute of Mathematical Sciences, Chennai 600 113, India \\ ${ }^{3}$ Center for Theoretical Studies, Indian Institute of Science, Bangalore 560 012, India
}

Received 8 May 2001, in final form 16 July 2001

Published 17 August 2001

Online at stacks.iop.org/JPhysA/34/6553

\begin{abstract}
A modified Gross-Pitaevskii approximation was recently introduced for bosons in dimension $d \leqslant 2$ by Kolomeisky et al (Kolomeisky E B, Newman T J, Straley J P and Qi X 2000 Phys. Rev. Lett. 85 1146). We use the densityfunctional approach with a sixth-degree interaction energy term in the Bose field to reproduce the stationary-frame results of Kolomeisky et al for a onedimensional Bose-Einstein system with a repulsive interaction. We also find a soliton solution for an attractive interaction, which may be boosted to a finite velocity by a Galilean transformation. The stability of such a soliton is discussed analytically. We provide a general treatment of stationary solutions in one dimension which includes the above solutions as special cases. This treatment leads to a variety of stationary wave solutions for both attractive and repulsive interactions.
\end{abstract}

PACS numbers: 03.75.Fi, 05.30.Jp

\section{Introduction}

There has been a number of theoretical studies on Bose-Einstein condensation (BEC) in a trap that is effectively lower-dimensional [1-8]. A three-dimensional (axially symmetric) anisotropic harmonic trap may be regarded as (quasi) two- or one-dimensional when $\omega_{\perp} \ll \omega_{x}$, or $\omega_{\perp} \gg \omega_{x}$ respectively, at temperatures much smaller than the larger of the oscillator energy gaps. Recently, Burger et al [9] experimentally created dark solitons in such a quasi onedimensional system of dilute $\mathrm{Rb}^{87}$ atoms. In this experiment, $\omega_{\perp}$ was taken to be about 30 times larger than $\omega_{x}$. After the creation of the soliton by phase-imprinting on the cigar-shaped condensate, the trap is switched off, and the soliton is observed to propagate along the $x$ direction. The s-wave interatomic interaction between $\mathrm{Rb}^{87}$ atoms is repulsive, and stable dark solitons are expected to exist in one dimension, as seen in this experiment. We note, however, that for $\mathrm{Rb}^{85}$ and $\mathrm{Li}^{7}$, the interatomic interaction is attractive, and bright solitons may form. 
Such solitons have been studied for the quasi one-dimensional case by several groups [4, 6], using Gross-Pitaevskii (GP) mean-field theory [10]. The standard GP theory replaces the bosonic-field operator by a classical-order parameter field $\Phi(\boldsymbol{r}, t)$ in three dimensions, and the potential energy is given by a fourth-degree $|\Phi|^{4}$ term in the energy-density functional. PerezGarcia et al [4] have shown how this may be reduced, for extreme anisotropy $\left(\omega_{\perp} \gg \omega_{x}\right)$, to the quasi one-dimensional geometry with a fourth-degree potential-energy term. It has also been shown that for the one-dimensional case with this attractive fourth-degree interaction potential, the solitons are dynamically stable (for the repulsive case, this has been known for a long time [11]). One has also considered the limiting case for $\omega_{x}=0$, when there is no confinement in the $x$-direction, and studied the free propagation of the soliton.

A different approach has been taken recently by Kolomeisky et al [12]. Instead of starting with the usual three-dimensional GP energy functional and reducing it to the quasi onedimensional case as described above, they have derived the GP functional for truly lower dimensions, $d \leqslant 2$, using the renormalization group approach [13]. In particular, they proposed, for a repulsive interatomic interaction, a sixth-degree potential-energy term replacing the usual fourth-degree term in the order parameter. With this modification they obtained a (dark) soliton solution in the repulsive case.

In this paper, we first discuss the solutions of the modified GP formalism in one dimension in the stationary frame. We show that the soliton solution of Kolomeisky et al [12] may be reproduced in the stationary frame with a repulsive interaction. Since the number of particles (see the definition in the next section) is generally not finite unless regulated, the phase cannot be obtained by a Galilean boost even though the system has Galilean invariance to begin with.

Next we focus on an attractive sixth-degree interaction term in the energy functional for bosons. Unlike the repulsive sixth-degree interaction, the attractive interaction may not be obtainable as a modified GP functional in one dimension. However, the motivations for taking such a phenomenological attractive potential-energy functional are two-fold. In three dimensions, using the standard fourth-degree attractive interaction energy term in the GP functional, it was predicted that the soliton-like solution would be stable only up to a limited number of bosons in the condensate [2,14-16], and this was verified experimentally [17]. Of course, in one dimension with an attractive fourth-degree term, the soliton is variationally stable as we will show in section 3. We want to examine the analytical solutions for the attractive sixth-degree interaction in the one-dimensional case. Analytical solutions for a more general case which includes the sixth-degree interaction were derived earlier in a different context by Pelinovsky et al [18]. They have also discussed numerically the stability of these solutions. Here we use the density-functional approach to derive these analytical solutions in the stationary frame. We show that there exists a limitation on the particle number for stability. Since the particle number is finite in the attractive case, we may use the Galilean invariance to obtain the general solution in an arbitrary frame.

It should also be mentioned that the fourth- and sixth-degree interaction (in the field variable) in the energy functional has been used extensively in various applications of physics [19], with a recent one [20] examining the stability of the $\mathrm{Li}^{7}$ condensate with an attractive two-body and a repulsive three-body contact interaction in three dimensions. More recently, the stability of an attractive BEC in the presence of a sixth-degree interaction with confinement has been analysed by Zhang [21]. The purely sixth-degree energy functional in one dimension, without the confining potential considered by Kolomeisky et al [12], is special because it is scale-invariant, and has a peculiar condition on the particle number for the soliton in the attractive case. We further show that there are soliton as well as stationary wave solutions if one uses the phenomenological model for attractive interaction. 
In section 2, we discuss the equations of motion in the stationary frame and their symmetries. In section 3, we obtain the solutions for repulsive and attractive interactions, and discuss the stability of the soliton solution in the attractive case. Section 4 provides a general discussion of the stationary solutions which includes all the above solutions as special cases.

\section{Equations of motion}

Our starting point is equations (3) and (4) of Kolomeisky et al [12] without a trap potential. The modified GP functional for a one-dimensional Bose-Einstein system is given by

$$
E=\frac{\hbar^{2}}{2 m} \int \mathrm{d} x\left[\frac{\mathrm{d} \Phi}{\mathrm{d} x} \frac{\mathrm{d} \Phi^{*}}{\mathrm{~d} x}+\frac{g \pi^{2}}{3}\left(\Phi \Phi^{*}\right)^{3}\right] .
$$

The first term is the kinetic-energy density and the second term arises from interactions; here the dimensionless coupling $g$ may be positive or negative. Note that the interaction term in (1) for the density $\rho=\Phi \Phi^{\star}$ is cubic and not quadratic as in the standard theory. A rigorous derivation of this term for the repulsive case may be found in [13]. It suffices here to state that in one dimension, where particles are not allowed to cross, such a term naturally arises when the interaction is short-range. This has been demonstrated for the hard-core interaction by Girardeau [22] in the thermodynamic limit, and by Lieb and Liniger [23] for a repulsive deltafunction potential. Even with a two-body interaction which varies inversely as the distance between particles, the leading term in the density functional has a cubic dependence to the density [24].

A few remarks may be made here in connection with the comment made by Bhaduri and Sen [25] recently. The Lieb and Liniger analysis [23] yields the result that in the limit of very low density of very strong repulsive interaction, the system becomes fermionic with $g=1$ in the functional given in equation (1). In the ground state, the density is constant and the kinetic term vanishes. Hence one obtains the free fermion result for the energy per unit length

$$
E=\frac{\hbar^{2}}{2 m} \frac{\pi^{2}}{3} \rho^{3} \text {. }
$$

Obviously, in the free fermion limit no soliton solutions occur. In what follows, we keep both the kinetic term and the interaction term between the bosons with a general coupling $g$, and therefore are not in the free fermion limit.

The equation of motion may be obtained from the energy functional in equation (1), and is given by

$$
\frac{\hbar^{2}}{2 m}\left[-\frac{\partial^{2}}{\partial x^{2}}+g \pi^{2}|\Phi|^{4}\right] \Phi(x, t)=\mathrm{i} \hbar \frac{\partial \Phi(x, t)}{\partial t} .
$$

Henceforth we shall denote the partial derivatives with respect to $x$ by a prime and with respect to $t$ by a dot. It is useful to multiply equation (3) by $\Phi^{*}(x, t)$

$$
-\frac{\hbar^{2}}{2 m} \Phi^{*} \Phi^{\prime \prime}+\Phi^{*} W(x) \Phi=\mathrm{i} \hbar \Phi^{*} \dot{\Phi}
$$

and take the complex conjugate

$$
-\frac{\hbar^{2}}{2 m} \Phi \Phi^{* \prime \prime}+\Phi W(x) \Phi^{*}=-\mathrm{i} \hbar \Phi \dot{\Phi}^{*}
$$

where $W(x)=\left(\hbar^{2} / 2 m\right) g \pi^{2}|\Phi|^{4}$. 
We now set, quite generally

$$
\Phi(x, t)=\sqrt{\rho(x, t)} \mathrm{e}^{\mathrm{i} \theta(x, t)}
$$

where $\rho$ and $\theta$ are real. It is useful to define the total particle number

$$
N=\int \mathrm{d} x \rho
$$

and the momentum functional

$$
P=\frac{-\mathrm{i} \hbar}{2} \int \mathrm{d} x\left[\Phi^{*} \Phi^{\prime}-\Phi^{* \prime} \Phi\right]=\hbar \int \mathrm{d} x \rho \theta^{\prime} .
$$

We then obtain the equations of motion as follows. The difference between equations (4) and (5) reduces to the continuity equation for the number density $\rho$

$$
\dot{\rho}+\frac{\hbar}{m}\left(\rho \theta^{\prime}\right)^{\prime}=0
$$

Thus it is legitimate to regard the velocity of the 'fluid' to be

$$
v(x, t)=\frac{\hbar}{m} \theta^{\prime}(x, t) .
$$

The energy-density equation is obtained by adding equations (4) and (5) and dividing by 2

$$
\tau(x, t)+W(x) \rho=-\hbar \rho \dot{\theta}
$$

where the kinetic-energy density is

$$
\begin{aligned}
\tau(x, t) & =-\frac{\hbar^{2}}{4 m}\left(\Phi^{*} \Phi^{\prime \prime}+\Phi^{* \prime \prime} \Phi\right) \\
& =\frac{\hbar^{2}}{2 m}\left(\frac{1}{4} \frac{\left(\rho^{\prime}\right)^{2}}{\rho}-\frac{1}{2} \rho^{\prime \prime}+\rho\left(\theta^{\prime}\right)^{2}\right) .
\end{aligned}
$$

Note that equations (9) and (11) are invariant under scaling and Galilean boosts [24] Under scaling by a factor $\alpha$

$$
\begin{aligned}
& \rho(x, t) \rightarrow \alpha \rho\left(\alpha x, \alpha^{2} t\right) \\
& \theta(x, t) \rightarrow \theta\left(\alpha x, \alpha^{2} t\right) .
\end{aligned}
$$

Then the particle number $N$ remains invariant, while the energy and momentum transform as

$$
E \rightarrow \alpha^{2} E \quad \text { and } \quad P \rightarrow \alpha P .
$$

Under a Galilean boost by velocity $v$

$$
\begin{aligned}
& \rho(x, t) \rightarrow \rho(x-v t, t) \\
& \theta(x, t) \rightarrow \theta(x-v t, t)+\frac{m v}{\hbar}\left(x-\frac{1}{2} v t\right) .
\end{aligned}
$$

Then the particle number $N$ remains invariant while the energy and momentum transform as

$$
\begin{aligned}
& E \rightarrow E+P v+\frac{1}{2} m N v^{2} \\
& P \rightarrow P+m N v .
\end{aligned}
$$

We should mention a subtlety here. If we are interested in solutions involving a single localized object (such as the solitons discussed in the next section), then it is reasonable to demand that its energy and momentum should only change by a finite amount under a boost. From equation (16), we see that this is true only if the particle number $N$ is finite. Therefore we will perform boosts only when $N$ is finite. 


\section{Stationary solutions: some analytical results}

We will now find some stationary solutions of equations (9) and (11). For such solutions we make the following ansatz:

$$
\Phi(x, t)=\sqrt{\rho(x)} \exp (-\mathrm{i} \mu t / \hbar)
$$

namely, we set $\theta(x, t)=-\mu t / \hbar$. Note that this ansatz is consistent with the continuity equation since both $\dot{\rho}$ and $\theta^{\prime}$ are zero. The equation for $\rho(x)$ is then given by

$$
\mu \rho=\frac{\hbar^{2}}{2 m}\left(\frac{1}{4} \frac{\left(\rho^{\prime}\right)^{2}}{\rho}-\frac{1}{2} \rho^{\prime \prime}+g \pi^{2} \rho^{3}\right) .
$$

Then the following solutions exist.

\subsection{Thomas-Fermi limit}

Assuming the bulk density to be a constant we set $\rho(x)=\rho_{0}$. This is an allowed solution with

$$
\rho_{0}=\sqrt{\frac{2 m \mu}{g \pi^{2} \hbar^{2}}}
$$

provided $g$ is positive. This is the Thomas-Fermi result [12]. Computing the energy, we reproduce the result given in equation (2) when $g=1$.

\subsection{Repulsive interaction}

We assume $g$ is positive and look for solutions when the density is not a constant. The Kolomeisky et al solution given in their equation (12) may be written in the stationary frame with their velocity parameter $\beta=0$ [12]. The solution is given by

$$
\rho(x)=\frac{k}{\pi \sqrt{g}} \frac{\cosh (2 k x)-1}{\cosh (2 k x)+2}
$$

with

$$
\mu=\frac{\hbar^{2} k^{2}}{2 m} .
$$

Note that the density tends to $\rho_{0}=1$ at $x \rightarrow \pm \infty$; hence it is better to define the particle number of the soliton to be

$N_{\text {soliton }}=\int \mathrm{d} x\left[\rho_{0}-\rho(x)\right]=\frac{3}{2 \pi \sqrt{g}} \int_{-\infty}^{\infty} \frac{\mathrm{d} t}{\cosh t+2}=\frac{\sqrt{3}}{\pi \sqrt{g}} \ln \left[\frac{\sqrt{3}+1}{\sqrt{3}-1}\right]$.

In nonlinear optics this number corresponds to the total intensity of light with a constant background subtracted out. Since $N_{\text {soliton }}$ is related to $g$, there exists exactly one solution for the system for a given value of $g$ (up to a scaling of $k$ ).

The above solution is called a dark soliton in optics [26] because the density goes to zero at $x=0$, and the soliton physically corresponds to a dip in the density distribution. Note that the above solution is valid when $g$ is positive, i.e., for repulsive interactions.

One can get the energy, $E$ of the soliton by integrating the energy density [27] given in equation (1). Using the soliton profile given in equation (20) and using equation (18) it is easy to see that the energy of the soliton is infinite. However if the energy of the soliton is defined as the difference

$$
E_{\text {soliton }}=E-E_{0}
$$


where $E_{0}$ is the energy of the solution with $\rho(x)=\rho_{0}=k / \pi \sqrt{g}$, then it turns out that the energy of the soliton is zero. It is interesting that even in the presence of a repulsive interaction, it does not cost any energy to create a soliton.

Until now we have checked the results given in [12] in the stationary frame. Next we consider some new solutions in the presence of an attractive interaction.

\subsection{Attractive interaction}

The equation of motion for $\Phi(x, t)$ in the stationary frame for $\rho$ may also be solved by the following ansatz

$$
\rho=\frac{2 k N}{\pi} \operatorname{sech}(2 k x) \quad \text { and } \quad \theta=-\frac{\mu t}{\hbar} .
$$

Note that the density is normalized so that

$$
\int_{-\infty}^{\infty} \mathrm{d} x \rho(x)=N
$$

The ansatz in (24) satisfies the equation of motion provided that

$$
N^{2}=-\frac{3}{4 g} \quad \text { and } \quad \mu=-\frac{\hbar^{2} k^{2}}{2 m} .
$$

Since $N$ is real we are forced to choose $g$ to be negative. We may therefore write

$$
N^{2}=\frac{3}{4|g|} .
$$

Following [26] we call this the bright soliton. We note in passing that a spatial soliton of the secant hyperbolic type is well known in nonlinear optics, and was experimentally realized long ago [28], although the differential equation obeyed by the solitonic-electrical field in optics is very different from ours.

Since $N$ and $g$ are related to each other by equation (27), there exists exactly one solution (up to scaling) for the system given a value for either $g$ or $N$. The soliton solution in this case is a lump, and has its maximum at $x=0$. Note the peculiar fact that the larger the magnitude of $g$, the smaller is the particle number $N$ of the soliton. The reason for this is explained in the next subsection where we will show that a configuration whose particle number is too large is unstable to collapse.

Interestingly, we find that the energy of the soliton in this case is zero. Since $N$ is finite, we can use equation (15), to boost the solution given in equation (24). Thus

$$
\begin{aligned}
& \rho=\frac{2 k N}{\pi} \operatorname{sech}(2 k(x-v t)) \\
& \theta=-\frac{\mu t}{\hbar}+\frac{m v}{\hbar}\left(x-\frac{1}{2} v t\right) .
\end{aligned}
$$

We then find that

$$
E=\frac{1}{2} m N v^{2} \quad \text { and } \quad P=m N v .
$$

Hence it is natural to define the mass of this soliton to be $M=m N$.

We further note that there also exist stationary wave solutions apart from the above. Starting from the dark soliton solution given in equation (20), we can transform $k \rightarrow \mathrm{i} k$ and $\rho \rightarrow-\rho$ to obtain the following stationary wave solution for negative values of $g$ :

$$
\rho(x)=\frac{k}{\pi \sqrt{-g}} \frac{1-\cos (2 k x)}{2+\cos (2 k x)}
$$


with

$$
\mu=-\frac{\hbar^{2} k^{2}}{2 m}
$$

Finally, note that we can apply the scaling transformation given in equation (13) for all the solutions given above; that would change the normalization of the density in all cases. But we can apply the boost transformation only for the bright soliton since that is the only case in which the total particle number is finite.

\subsection{Stability of the soliton solutions}

We will now study the stability of the solitons, particularly the bright soliton given in equation (24), using both variational and small fluctuation analysis. Before doing that, let us make some preliminary comments.

The soliton solutions obtained above for both attractive and repulsive cases are scale invariant. Hence the type of instability which is associated with the soliton solutions for certain types of nonlinear Schrödinger equations (see [29]) are not expected here. Another form of instability which can occur is the breaking of the soliton into some other fundamental constituents [30]. To check the possibility of that, let us note that our energy functional has a global $U(1)$ symmetry, and hence a conserved charge associated with such a soliton solution. For the attractive case, the charge is given by the expression

$$
Q=\mathrm{i} \hbar \int \mathrm{d} x\left(\Phi^{\dagger} \dot{\Phi}-\dot{\Phi}^{\dagger} \Phi\right)=2 \mu N=-\frac{\hbar^{2} k^{2}}{2 m} \sqrt{\frac{3}{|g|}} .
$$

This is of course proportional to the integral of the density $\rho(x)$ in our case which is the same in any frame. In that case the soliton solution is classically stable against the aforesaid complete dissociation into more fundamental constituents ( $\mathrm{mfc}$ ), because its rest energy (which is 0 here) must be lower than $m_{0}|Q|$ if we assume that the mass $m_{0}$ of the hypothetical mfc must be positive.

We will now show that in the attractive case $g<0$, the bright soliton can collapse to a point if the particle number of a configuration is bigger than a critical number $N_{\mathrm{c}}$ which is a function of the parameter $g$. We can show this as follows. Consider a trial wavefunction of the form

$$
\Phi(x)=\left(\frac{N \alpha}{\pi \cosh (\alpha x)}\right)^{1 / 2}
$$

where $\alpha$ is a free parameter (whose inverse determines the width of the configuration), and we set $\theta=0$. The particle number for this configuration is given by $N$. We then find that the energy functional in equation (1) is given by

$$
E=\frac{\alpha^{2} \hbar^{2} N}{16 m}\left[1-\frac{4}{3}|g| N^{2}\right]
$$

for this configuration. We observe that this expression does not have a minimum at any finite and nonzero value of $\alpha$. If

$$
N^{2}>N_{\mathrm{c}}^{2} \equiv \frac{3}{4|g|}
$$

then the energy of the configuration given in equation (33) can be made arbitrarily large and negative by letting $\alpha \rightarrow \infty$; this corresponds to the density of the configuration collapsing to a $\delta$-function at the origin. We thus see that there is a critical value of $N_{\mathrm{c}}$ which is proportional 
to $1 / \sqrt{|g|}$ beyond which there is no lower bound to the energy. We observe that the particle number of the bright soliton in equation (27) is exactly equal to this critical value. Since this argument is only based on a trial wavefunction, it does not prove anything about the stability of the bright soliton against a collapse.

It is instructive to contrast the situation here with a more general form of the GP functional where the interaction term in equation (1) is taken to be of the form $g \rho^{n}$ instead of $g \rho^{3}$, where we assume $n>1$. If the exponent of $\rho$ is taken to be $n<3$ (instead of $n=3$ as we have considered so far), and $g$ is negative, we find from the above argument that there is no instability to a collapse, no matter what the values of $g$ and $N$ are. Namely, for any value of $g<0$ and $N$, the energy of the trial configuration given in (33) has a minimum at some finite value of the inverse width $\alpha$. On the other hand, if we take the exponent $n>3$ and $g<0$, the above argument indicates an instability to collapse for any value of $N$, no matter how small. Thus, an attractive interaction of the form $\rho^{3}$ is rather special; this is the only value of the exponent $n$ for which there is a finite critical number $N_{\mathrm{c}}$, such that the trial configuration is stable against a collapse if $N<N_{\mathrm{c}}$ and is unstable if $N>N_{\mathrm{c}}$. This special property of $n=3$ can be traced to the fact that the coefficient of the interaction $g$ is dimensionless only for this value of $n$.

Let us now return to the bright soliton given in equation (24), and directly study its stability by studying small fluctuations around it. Our main conclusion will be that there is at least one unstable mode. To do the analysis, let us set $k=1 / 2$ for simplicity in (24). Then the soliton takes the form

$$
\begin{aligned}
\rho_{\mathrm{c}} & =\frac{N}{\pi \cosh x} \\
\theta_{\mathrm{c}} & =\frac{\hbar t}{8 m}
\end{aligned}
$$

with $N^{2} g=-3 / 4$. We now assume small fluctuations of the form

$$
\begin{aligned}
& \rho=\frac{N}{\pi \cosh x}+\delta \rho(x, t) \\
& \theta=\frac{\hbar t}{8 m}+\delta \theta(x, t) .
\end{aligned}
$$

The equations of motion then imply that

$$
\begin{aligned}
\frac{\partial \delta \rho}{\partial t} & =-\frac{\hbar N}{\pi m}\left(\frac{1}{\cosh x} \frac{\partial^{2}}{\partial x^{2}}-\frac{\sinh x}{\cosh ^{2} x} \frac{\partial}{\partial x}\right) \delta \theta \\
\frac{\partial \delta \theta}{\partial t} & =\frac{\pi \hbar}{4 N m} \cosh x\left(\frac{\partial^{2}}{\partial x^{2}}+\frac{\sinh x}{\cosh x} \frac{\partial}{\partial x}+\frac{\sinh ^{2} x}{2 \cosh ^{2} x}+\frac{9}{2 \cosh ^{2} x}-\frac{1}{2}\right) \delta \rho .
\end{aligned}
$$

To solve these equations, we eliminate $\delta \theta$ and define a function $\psi(x)$ as

$$
\delta \rho(x, t)=(\cosh x)^{-1 / 2} \psi(x) \mathrm{e}^{-\mathrm{i} \omega t} .
$$

We then obtain the eigenvalue equation

$$
A \psi(x)=\omega^{2} \psi(x)
$$

where

$$
A=\frac{\hbar^{2}}{4 m^{2}}\left(\frac{\mathrm{d}^{2}}{\mathrm{~d} x^{2}}-\frac{1}{4}+\frac{3}{4 \cosh ^{2} x}\right)\left(\frac{\mathrm{d}^{2}}{\mathrm{~d} x^{2}}-\frac{1}{4}+\frac{15}{4 \cosh ^{2} x}\right) \psi
$$

The operator $A$ is Hermitian with the weight $w(x)=1$; namely, for any two normalizable functions $\psi_{1}(x)$ and $\psi_{2}(x)$

$$
\int_{-\infty}^{\infty} \mathrm{d} x w(x) \psi_{1}^{\star}(x) A \psi_{2}(x)=\int_{-\infty}^{\infty} \mathrm{d} x w(x)\left(A \psi_{1}(x)\right)^{\star} \psi_{2}(x)
$$


with $w(x)=1$. (It is for this reason that we introduced the factor of $(\cosh x)^{1 / 2}$ in the definition of $\psi$ in equation (39).) Hence the eigenvalues of $A, \omega^{2}$, must be real. However, $\omega^{2}$ could be negative; in that case, there would be a solution in which $\omega$ is purely imaginary and $-\mathrm{i} \omega>0$. That would be an unstable mode with the function $\delta \rho(x, t)$ blowing up exponentially as $t$ goes to $\infty$. We therefore need to know if $A$ has any negative eigenvalues.

We already know two eigenfunctions of $A$ with $\omega=0$ corresponding to the translation and scaling symmetries. From infinitesimal versions of these two symmetries, we find that the eigenfunctions of the operator $A$ with zero eigenvalues are given by

$$
\psi=\frac{\sinh x}{(\cosh x)^{3 / 2}}
$$

and

$$
\psi=\frac{1}{(\cosh x)^{1 / 2}}-\frac{x \sinh x}{(\cosh x)^{3 / 2}} .
$$

Note that these two functions are normalizable (i.e., they would be called bound states in the language of quantum mechanics), and that they have one and two nodes, respectively. Thus the operator $A$ has quite different properties from the usual Hamiltonian operator $H=-\mathrm{d}^{2} / \mathrm{d} x^{2}+V(x)$ which appears in a one-dimensional Schrödinger equation; a second derivative operator like $H$ always has non-degenerate eigenvalues for bound states, and the corresponding eigenvalues strictly increase with the number of nodes.

We have not been able to find an analytical expression for the eigenfunction of $A$ corresponding to an eigenvalue $\omega^{2}<0$. However, it is possible to show its existence by a variational argument. We use the result from quantum mechanics that for a Hermitian operator $A$, if $\psi_{v}(x)$ is a normalizable variational function, and

$$
E_{v}=\frac{\left\langle\psi_{v}|A| \psi_{v}\right\rangle}{\left\langle\psi_{v} \mid \psi_{v}\right\rangle}
$$

is the corresponding variational energy, then there must be an eigenvalue of $A$ which is less than or equal to $E_{v}$. We now try a function of the form

$$
\psi_{\alpha}(x)=\frac{1}{(\cosh x)^{1 / 2}}+\alpha \frac{x \sinh x}{(\cosh x)^{3 / 2}}
$$

where $\alpha$ is a variational parameter which we assume to be real for convenience. Then the variational energy given in (44) takes the form

$$
E_{\alpha}=\frac{3 \hbar^{2}}{10 m^{2}} \frac{\alpha(1+\alpha)}{1+2 \alpha+\alpha^{2}\left(1+\pi^{2} / 8\right)} .
$$

We find numerically that this has a minimum value of $E=-0.1350 \hbar^{2} / \mathrm{m}^{2}$ at $\alpha=-0.4738$. Since this minimum value is negative, we know that there must be an eigenfunction of the operator $A$ for which $\omega^{2}<0$. This shows that the bright soliton has at least one small fluctuation mode which blows up as $t \rightarrow \infty$. Since we do not know the explicit form of this eigenfunction, the physical interpretation of this mode remains unclear.

Although we have found an instability classically, it is possible that the instability may disappear if the theory is quantized, i.e., if we impose the equal-time commutation relation $[\rho(x), \theta(y)]=\mathrm{i} \delta(x-y)$ [24]. There are examples known in the literature (for instance, skyrmions in three dimensions) where the quantization of some collective coordinates eliminates a classical instability [31]. However, we will not examine the question of the quantum stability of the bright soliton in this paper where our entire analysis has been classical. 


\section{General treatment of stationary solutions}

We will now see how the general form of the stationary solution of equation (3) may be found. We do this by mapping the one-dimensional problem considered in previous sections to that of a pseudo-particle problem in two dimensions. To do this let us write

$$
\Phi(x, t)=\mathrm{e}^{-\mathrm{i} \mu t / \hbar}\left[\xi_{1}(x)+\mathrm{i} \xi_{2}(x)\right] .
$$

Thus the density given by $\rho=\xi_{1}^{2}+\xi_{2}^{2}$ is independent of time. Equation (3) then takes the form

$$
\begin{aligned}
\frac{\mathrm{d}^{2} \xi_{1}}{\mathrm{~d} x^{2}} & =-\frac{2 m \mu}{\hbar^{2}} \xi_{1}+g \pi^{2} \rho^{2} \xi_{1} \\
\frac{\mathrm{d}^{2} \xi_{2}}{\mathrm{~d} x^{2}} & =-\frac{2 m \mu}{\hbar^{2}} \xi_{2}+g \pi^{2} \rho^{2} \xi_{2} .
\end{aligned}
$$

These are precisely the equations of motion of a classical particle of unit mass moving in two dimensions with the spatial coordinates $\left(\xi_{1}, \xi_{2}\right)$ and a time coordinate $x$. Note that in the one-dimensional problem $x$ refers to position coordinate. The total energy and pseudo-angular momentum of the particle are given by

$$
\begin{aligned}
& \mathcal{E}=\frac{1}{2}\left(\frac{\mathrm{d} \xi_{1}}{\mathrm{~d} x}\right)^{2}+\frac{1}{2}\left(\frac{\mathrm{d} \xi_{2}}{\mathrm{~d} x}\right)^{2}+\frac{m \mu}{\hbar^{2}} \rho-\frac{g \pi^{2}}{6} \rho^{3} \\
& L=\xi_{1} \frac{\partial \xi_{2}}{\partial x}-\xi_{2} \frac{\partial \xi_{1}}{\partial x} .
\end{aligned}
$$

The pseudo-angular momentum $L$ is in fact the same, up to a factor, as the momentum density given in equation (8). These are conserved since the particle is moving in a central potential

$$
V\left(\xi_{1}, \xi_{2}\right)=\frac{m \mu}{\hbar^{2}} \rho-\frac{g \pi^{2}}{6} \rho^{3}
$$

Since there are two conserved quantities and the particle has two degrees of freedom, the motion can be found exactly if the initial conditions are given. For instance, $\rho$ is given in terms of $x$ by the equation

$$
\left(\frac{\mathrm{d} \rho}{\mathrm{d} x}\right)^{2}=4\left[2 \rho \mathcal{E}-L^{2}-\frac{2 m \mu}{\hbar^{2}} \rho^{2}+\frac{g \pi^{2}}{3} y \rho^{4}\right] .
$$

We are interested in solutions of equations (48) and (51) in which the particle density $\rho$ remains finite as $x \rightarrow \pm \infty$. In that case, the solution for $\rho$ will generally be a periodic function of $x$; this corresponds to a stationary wave. From the form of the potential energy in (49), we see that this will happen in the following situations.

(i) If $g>0$, the potential has a negative sixth-degree term. Hence we must have $\mu>0$ and the particle energy $\mathcal{E}$ must be equal to or less than the potential barrier (this can be derived from equation (50)) in order to have $\rho$ finite for all times $x$. Then $\rho$ will always remain bounded by the location of the potential barrier given by $\rho_{0}=\sqrt{2 m \mu / g \pi^{2} \hbar^{2}}$ as in equation (19).

(ii) If $g<0$, the potential has a positive sixth-degree term, so $\rho$ will be finite regardless of whether $\mu$ is positive or negative. However the possible motions are different for $\mu>0$ and $\mu<0$ as we will see.

We will now discuss how the various solutions discussed in the previous section can be obtained as special cases from this general discussion. First of all, let us set the pseudo-angular momentum $L=0$ and therefore $\xi_{2}=0$. Let us now consider the cases with $g$ positive and negative separately. 
(i) In the repulsive case $g>0$, we have already seen that we need $\mu>0$ and the energy must lie below the potential barrier so that the particle does not escape to infinitely large values of $\rho$. If the energy is exactly equal to the potential barrier, then we have two possible solutions. Either the particle stays at the top of the potential barrier for all times $x$ (this corresponds to the Thomas-Fermi result in (19)), or we have a solution in which the particle begins at $\xi_{1}=-\sqrt{\rho_{0}}$ at time $x=-\infty$ and ends at $\xi_{1}=\sqrt{\rho_{0}}$ at time $x=\infty$. The latter solution corresponds to the dark soliton of Kolomeisky et al. On the other hand, if the energy is less than the potential barrier, then the particle will perform a periodic motion which repeatedly passes through $\xi_{1}=0$; this corresponds to a stationary wave with the minimum density being zero at an infinite number of values of $x$. This is a new stationary wave solution.

(ii) In the attractive case $g<0$, there is only one kind of motion possible if $\mu>0$, i.e. the particle will perform a periodic motion passing repeatedly through $\xi_{1}=0$, thus giving a new stationary wave. If $\mu<0$, we have a double-well potential (see equation (50)), and there are various kinds of motion possible depending on the energy $\mathcal{E}$. If $\mathcal{E}>0$, then the particle again performs a periodic motion passing through $\xi_{1}=0$, thereby giving the stationary wave described in section 2.3. If $\mathcal{E}=0$, then we have a bounce solution in which the particle begins at $\xi_{1}=0$ at $x=-\infty$, goes to some maximum (or minimum) value of $\xi_{1}$ at some finite time and then returns to $\xi_{1}=0$ at $x=\infty$. This corresponds to the bright soliton described in section 3.3; note that such a solution necessarily has $N^{2} g=-3 / 4$ no matter what the value of $\mu$ is. (Thus there is no bright soliton solution possible if $N^{2} g \neq-3 / 4$.) If $\mathcal{E}$ is negative but is greater than the bottom of the potential, the particle performs a periodic motion in which $\xi_{1}$ always remains positive (or negative) and never reaches zero. This is a new solution corresponding to a stationary wave whose minimum density is nonzero. Finally, if $\mathcal{E}$ is equal to the bottom of the potential located at $\rho_{0}=\sqrt{2 m \mu / g \pi^{2} \hbar^{2}}$, then we have a solution with uniform density $\rho(x)=\rho_{0}$.

\section{Comments}

We have shown that the modified one-dimensional GP mean-field theory has many solutions apart from the one outlined by Kolomeisky et al [12]. The soliton solution in the attractive case is particularly simple since the full time-dependent solutions in an arbitrary moving frame may be obtained simply by boosting the solutions in the stationary frame. The question of the quantum stability of this soliton remains an open problem.

\section{Acknowledgments}

SG thanks R Rajaraman for discussions and comments. RKB acknowledges financial support from NSERC, Canada, for this research, and the hospitality of IMSc, Chennai, India.

\section{References}

[1] Bagnato V and Kleppner D 1991 Phys. Rev. A 447439

[2] Ruprecht P A, Holland M J, Burnett K and Edwards M 1995 Phys. Rev. A 514704

[3] Ketterle W and van Druten N J 1996 Phys. Rev. A 54656

[4] Perez-Garcia V M, Michinel H and Herrero H 1998 Phys. Rev. A 573837

[5] Muryshev A E, van Linden van den Heuvell H B and Shlyapnikov G V 1999 Phys. Rev. A 60 R2665

[6] Carr L D, Leung M A and Reinhardt W P 2000 J. Phys. B: At. Mol. Opt. Phys. 333983

[7] Petrov D S, Holzmann M and Shlyapnikov G V 2000 Phys. Rev. Lett. 842551

[8] Bhaduri R K, Reimann S M, Viefers S, Ghose Choudhury S and Srivastava M K 2000 J. Phys. B: At. Mol. Opt. Phys. 333895 
[9] Burger S et al 1999 Preprint cond-mat/9910487

[10] Pitaevskii L P 1961 Zh. Eksp. Teor. Fiz. 40646 (Engl. transl. 1961 Sov. Phys.-JETP 13 451)

Gross E P 1961 Nuovo Cimento 20454

[11] Zakharov V E and Shabat A B 1972 Sov. Phys.-JETP 3462

[12] Kolomeisky E B, Newman T J, Straley J P and Qi X 2000 Phys. Rev. Lett. 851146

[13] Kolomeisky E B and Straley J P 1992 Phys. Rev. B 4611749

[14] Dalfovo F and Stringari S 1996 Phys. Rev. A 532477

[15] Kagan Y, Shlyapnikov G V and Walraven J T M 1996 Phys. Rev. Lett. 762670

[16] Dodd R J et al 1996 Phys. Rev. A 54661

[17] Bradley C C, Sackett C A and Hulet R G 1997 Phys. Rev. Lett. 78985

[18] Pelinovsky D E, Afanasjev V V and Kivshar Y S 1996 Phys. Rev. E 531940

For a further discussion on stability, see also Berge L 2000 Phys. Rev. E 62 R3071

[19] Barashenkov I V, Puzynin I V and Zhanlav T 1990 4th Int. Workshop on Solitons and Applications (Aug. 1989) ed V G Makhankov, V K Fedyanin and O K Pashaev (Singapore: World Scientific) p 281 and references therein

[20] Akhmediev N, Das M P and Vagov A V 1999 Int. J. Mod. Phys. 13625

[21] Zhang J 2000 J. Stat. Phys. 101731

[22] Girardeau M 1960 J. Math. Phys. 1516

[23] Lieb E H and Liniger W 1963 Phys. Rev. 1301605

[24] Sen D and Bhaduri R K 1997 Ann. Phys., NY 260203

[25] Bhaduri R K and Sen D 2001 Phys. Rev. Lett. 864708

[26] Kivshar Y S and Luther-Davies B 1998 Phys. Rep. 29881

[27] Rajaraman R 1982 Solitons and Instantons (Amsterdam: North-Holland)

[28] Chiao R Y Introduction to spatial solitons Spatial Solitons ed S Trillo and W E Torruellas (New York: Springer) volume 82

Chiao R Y, Garmire E and Townes C H 1964 Phys. Rev. Lett. 13479

[29] Barashenkov I V and Makhankov V G 1988 Phys. Lett. A 12852

Barashenkov I V, Gocheva A D, Makhankov V G and Puzynin I V 1989 Physica D 34240

[30] Raj Lakshmi M 1979 Pramana 12447

[31] Abdalla A and Preston M A 1996 Phys. Rev. D 533967 